\title{
Long surgical waiting list times are associated with an increased rate of negative ureteroscopies
}

\author{
Daniel A. González-Padilla, MD'; Alejandro González-Díaz, MD²; Helena Peña-Vallejo, MD²; Rocío Santos \\ Pérez de la Blanca, MD2; Julio Teigell-Tobar, MD2; Mario Hernández-Arroyo, MD²; Pablo Abad-López, MD2; \\ Alfredo Rodriguez-Antolin, $M D^{2}$; Fernando Cabrera-Meiras, $M D^{2}$
}

'Department of Urology, University Hospital Infanta Sofía, Madrid, Spain; ${ }^{2}$ Department of Urology, University Hospital 12 de Octubre, Madrid, Spain

\begin{abstract}
Cite as: González-Padilla DA, González-Díaz A, Peña-Vallejo H, et al. Long surgical waiting list times are associated with an increased rate of negative ureteroscopies. Can Urol Assoc J 2021;15(12): 407-11. http://dx.doi.org/10.5489/cuaj.7199
\end{abstract}

Published online June 22, 2021

See related commentary on page 412

\section{Abstract}

Introduction: Negative ureteroscopy (NURS) is "a ureteroscopy in which no stone is found during the procedure." We aimed to determine the association between the surgical waiting list time (WLT) and the NURS rate.

Methods: We retrospectively analyzed all patients scheduled for ureteroscopy in our center between January 2017 and July 2019 The inclusion criterion was unilateral, semirigid ureteroscopy for a single ureteral stone; exclusion criteria were renal-only stones, incomplete ureteroscopy, and stones $>10 \mathrm{~mm}$. We analyzed age; gender; body mass index; stone size, density, and location; presence of a temporary double-J (DJ) stent; use of medical expulsive therapy; and WLT. Complications while waiting for surgery were also collected and analyzed.

Results: We included 219 patients, 41 (18.7\%) of whom had NURS. The median WLT was 74 days (interquartile range [IQR] 45-127). Variables protective against NURS were large stone size (odds ratio [OR] 0.78, 95\% confidence interval [CI] 0.66-0.93), presence of a temporary DJ stent (OR $0.43,95 \% \mathrm{Cl} 0.2-0.8)$, and radiopaque stones (OR $0.44,95 \% \mathrm{Cl} 0.21-0.88)$. A long WLT $(\geq 60$ days) increased the risk of NURS (OR 2.18, 95\% CI 1.02-4.61). Complications requiring emergency department visits while waiting for surgery were documented in 58/137 (42.3\%) patients with indwelling DJ stents; nonetheless, a WLT greater than the median was not associated with an increased risk of complications $(p=0.38)$.

Conclusions: Long WLT has an independent, direct, and linear correlation with NURS rates. Patients at higher risk of NURS, may be offered preoperative re-evaluation with a computed tomography scan in a resource-limited setting.

\section{Introduction}

Negative ureteroscopy (NURS), also called "stoneless" or "diagnostic" ureteroscopy, is defined as "a ureteroscopy in which no stone is found during the procedure" because the stone either has already been passed spontaneously or is located outside the collecting system.

The incidence of NURS has been reported in some series to be between $3.8 \%$ and $13 \% .^{1-5}$ These series have reported certain risk factors to be associated with NURS, including female gender, small stone size, low stone density, and distal location.

It is important to identify patients with an increased risk of NURS in order to develop successful pathways that may help prevent unnecessary surgical interventions, with their associated complications and economic costs.

Another factor to be taken into account is radiation exposure associated with computed tomography (CT) scan, which is the imaging modality of choice for the detection of urolithiasis; this exposure has been reported to be around 3 millisieverts (mSv) for low-dose CT and between 9.6 and 12.6 $\mathrm{mSv}$ for standard CT. ${ }^{6}$ Again, this highlights the importance of appropriate selection of patients at high risk of NURS for preoperative imaging.

The reasons for the variability of NURS rates among the published series are not known. One factor that has not been directly studied is the influence of surgical waiting list time (WLT), which may partially explain the variability among centers. In the present study, we aimed to investigate the influence of surgical WLT on NURS rates.

\section{Methods}

We conducted a retrospective cohort study analyzing all scheduled semirigid ureteroscopies performed in our department between January 2017 and July 2019. All patients who underwent scheduled, unilateral, semirigid ureteroscopy for a single ureteral stone with or without an indwelling double-J 
(D) stent were included. Exclusion criteria were renal-only stones, incomplete ureteroscopy due to inability to explore the entire length of the ureter, and stones larger than $10 \mathrm{~mm}$.

We analyzed age, gender, body mass index (BMI), stone density (Hounsfield units [HU]), stone size, stone location (upper, mid, or distal ureter), presence of a temporary DJ stent, use of medical expulsive therapy, and WLT. HU were measured in the CT scan whenever available. WLT was defined as the number of days between the date of the imaging test used as the basis for inclusion of patients on the waiting list (usually performed a few days before consultation and 4-6 weeks after initial presentation of the renal colic) and the date of surgery. The imaging test was always either a CT scan or a combination of kidney-ureter-bladder (KUB) X-ray and ultrasound (the stone had to be clearly visible on either test to warrant inclusion of the patient on the waiting list).

Stone size was assessed by measuring the largest diameter on the most precise imaging test available, with $\mathrm{CT}$ being the imaging modality of choice, followed by KUB X-ray, and finally ultrasound.

Stone location was defined as proximal (from the renal pelvis to the upper edge of the sacrum), mid (from the upper to the lower edge of the sacrum), or distal (from the lower edge of the sacrum to the urinary bladder). ${ }^{7}$

Patients were not routinely prescribed medical expulsive therapy while awaiting surgery.

Patients with temporary DJ stents had them placed due to prior complicated renal colic, sepsis, obstructive acute renal failure (defined as an increase in serum creatinine to $\geq 1.5$ times baseline, known or presumed to have occurred within the prior seven days), suspected concomitant urinary tract infection, single functioning kidney, or analgesic refractory colic pain.

By the time the data were collected, we were routinely performing KUB X-ray on the day before the procedure in all patients who were scheduled for semirigid ureteroscopy and had radiopaque stones; if the stone was radiolucent, imaging was omitted. If no stone was seen on the KUB X-ray and there was a history of possible spontaneous stone passage, the patient was offered cancellation of surgery with re-evaluation by means of a new CT scan or continuation of the scheduled surgery.

The surgery was performed with a semirigid ureteroscope until the renal pelvis was reached. If no stone was found, exploration of the renal pelvis and calyces was carried out with a flexible ureteroscope with or without a ureteral access sheath.

Complications associated with DJ stents (pain, hematuria, catheter-associated urinary tract infection, migration) while waiting for surgery were also documented and analyzed.

We followed the Strengthening the Reporting of Observational Studies in Epidemiology (STROBE) reporting guidelines from the EQUATOR network. ${ }^{8}$

\section{Statistical analysis}

Quantitative variables are presented by means and standard deviations or by medians and $25^{\text {th }}$ and $75^{\text {th }}$ percentiles. Qualitative variables are described according to the distribution of relative and absolute frequencies. Association between variables was explored with the Chi-squared test for categorical variables, while for continuous variables Student's t-test was used if a normal distribution was followed or the Wilcoxon-Mann-Whitney test if a normal distribution was not followed. The magnitude of association among variables was measured by the odds ratio (OR) with a 95\% confidence interval $(\mathrm{Cl})$. For the correlation between waiting list days and NURS, we used the receiver operator characteristics (ROC) curve and its characterization.

\section{Results}

We included 219 patients in the analysis, of whom 178 $(81.3 \%)$ had positive ureteroscopies (PURS) and 41 (18.7\%) had NURS. Mean age and BMI were similar in both groups, while a statistically significant difference in female gender prevalence was seen in the NURS group, with women accounting for $49 \%$ of this group compared to $36 \%$ in the PURS group ( $p=0.034)$. Regarding stone characteristics, stone density was similar between the two groups.

Distal stone location was more frequent in the NURS group, $73.1 \%$ vs. $54.5 \%$ in the PURS group, with the difference reaching statistical significance at univariate analysis $(p=0.048)$. In $137(62.5 \%)$ of the 219 patients, stones were measured using CT, while in $58(26.5 \%)$, the measurement was made using KUB X-ray, and in $24(11 \%)$ using ultrasound.

The median WLT in the whole cohort was 74 days (interquartile range [IQR] 45-127 days). Median WLT in the NURS group was longer than that in the PURS group, at 112 days vs. 67 days, with the difference reaching statistically significance at univariate analysis $(p=0.026)$. Demographic and clinical characteristics and the results of univariate analysis are summarized in Table 1.

In the multivariate analysis, four variables were found to be statistically significant, three of which were protective against NURS: large stone size (OR 0.78, 95\% Cl 0.66-0.93, $\mathrm{p}=0.006)$, presence of a temporary DJ stent (OR 0.43, 95\% $\mathrm{Cl} 0.2-0.8, \mathrm{p}=0.019$ ), and radiopaque stones (OR 0.44, 95\% CI 0.21-0.88, $\mathrm{p}=0.022)$. WLT increased the risk of NURS (OR 1.005, 95\% Cl 1.00-1.01) for each passing day, with the cumulative risk reaching OR 1.19 at 30 days and OR 2.18 at 60 days ( $p=0.024,0.024$, and 0.043 , respectively). These results are summarized in Table 2 .

A ROC curve analysis was performed for WLT in days and its ability to predict a NURS, with an area under the curve of 0.61 . A summary of the NURS rates per WLT by month is given in Table 3. 
Table 1. Demographic and clinical characteristics with univariate analysis

\begin{tabular}{|c|c|c|c|c|}
\hline & Total $(n=219)$ & Positive URS ( $n=178$ ) & Negative URS ( $n=41)$ & $\mathbf{P}$ \\
\hline Age (years), mean (range) & $57.6(19-89)$ & $57.6(19-89)$ & $57.9(22-86)$ & 0.39 \\
\hline $\begin{array}{l}\text { Gender, n (\%) } \\
\text { Male } \\
\text { Female }\end{array}$ & $\begin{array}{l}134(61.1 \%) \\
85(38.9 \%)\end{array}$ & $\begin{array}{l}113(63.5 \%) \\
65(36.5 \%)\end{array}$ & $\begin{array}{l}21(51 \%) \\
20(49 \%)\end{array}$ & $0.034 *$ \\
\hline BMI, mean (SD) & $28(4.7)$ & $28.3(4.9)$ & $26.9(3.7)$ & 0.14 \\
\hline Hounsfield units, median (IQR) & $744(528-910)$ & $736(563-924)$ & 679 (458-808) & 0.098 \\
\hline Stone size, mm, median (IQR) & $7(5-8)$ & $7(5-9)$ & $6(5-7)$ & $0.004^{*}$ \\
\hline $\begin{array}{l}\text { Location, n (\%) } \\
\text { Proximal } \\
\text { Mid } \\
\text { Distal }\end{array}$ & $\begin{array}{l}46(21 \%) \\
46(21 \%) \\
127(58 \%)\end{array}$ & $\begin{array}{l}42(23.6 \%) \\
39(21.9 \%) \\
97(54.5 \%)\end{array}$ & $\begin{array}{c}4(9.8 \%) \\
7(17.1 \%) \\
30(73.1 \%)\end{array}$ & $0.048 *$ \\
\hline $\begin{array}{l}\text { Preoperative DJ stent, n (\%) } \\
\text { No } \\
\text { Yes }\end{array}$ & $\begin{array}{c}82(37.5 \%) \\
137(62.5 \%)\end{array}$ & $\begin{array}{c}60(33.7 \%) \\
118(66.3 \%)\end{array}$ & $\begin{array}{l}22(53.7 \%) \\
19(46.3 \%)\end{array}$ & $0.020 *$ \\
\hline $\begin{array}{l}\text { Imaging test used, } \mathrm{n}(\%) \\
\text { Ultrasound + KUB } \\
\text { CT }\end{array}$ & $\begin{array}{c}82(37.5 \%) \\
137(62.5 \%)\end{array}$ & $\begin{array}{c}70(39.4 \%) \\
108(60.6 \%)\end{array}$ & $\begin{array}{l}12(29.3 \%) \\
29(70.7 \%)\end{array}$ & 0.30 \\
\hline Medical expulsive therapy use & $18(8.1 \%)$ & $14(7 \%)$ & $4(9.8 \%)$ & 0.75 \\
\hline $\begin{array}{l}\text { Radiopaque, n (\%) } \\
\text { No } \\
\text { Yes } \\
\text { Missing }\end{array}$ & $\begin{array}{c}67(30.6 \%) \\
148(67.6 \%) \\
4(1.8 \%)\end{array}$ & $\begin{array}{c}48(27 \%) \\
126(70.8 \%) \\
4(2.2 \%)\end{array}$ & $\begin{array}{c}19(46.3 \%) \\
22(53.7 \%) \\
0(0 \%)\end{array}$ & $0.025 *$ \\
\hline Waiting-list time, days, median (IQR) & $74(45-127)$ & $67(42-126)$ & $112(60-146)$ & $0.026 *$ \\
\hline
\end{tabular}

Complications associated with DJ stents that required emergency department visits while waiting for surgery were documented in 58 of the 137 patients (42.3\%) with indwelling DJ stents, seven of whom required DJ stent exchange in the operating room due to the complications (in two cases due to migration and in five due to sepsis). Nonetheless, a longer than the median WLT was not associated with an increased risk of complications $(p=0.38)$. Complications are summarized in Table 4.

\begin{tabular}{|c|c|c|}
\hline Risk factor & Odds ratio $(95 \% \mathrm{Cl})$ & $\mathbf{p}$ \\
\hline Gender (female) & $1.65(0.83-3.28)$ & $p=0.14$ \\
\hline BMI (per unit of $\mathrm{kg} / \mathrm{m}^{2}$ ) & $0.93(0.86-1.02)$ & $p=0.14$ \\
\hline Density (per HU) & $0.99(0.99-1.00)$ & $p=0.069$ \\
\hline Stone size $(\text { per } \mathrm{mm})^{\dagger}$ & $0.78(0.66-0.93)$ & $p=0.006^{*}$ \\
\hline Location (distal) & $1.8(0.8-4.3)$ & $p=0.14$ \\
\hline Preoperative DJ stent & $0.43(0.2-0.8)$ & $p=0.019 *$ \\
\hline Radiopaque & $0.44(0.21-0.88)$ & $\mathrm{p}=0.022 *$ \\
\hline Waiting list time (per day) & $1.005(1.00-1.01)$ & $\mathrm{p}=0.024^{*}$ \\
\hline Waiting list time (at 30 days) & $1.188(1.02-1.38)$ & $p=0.024^{*}$ \\
\hline Waiting list time (at 60 days) & $2.176(1.02-4.61)$ & $p=0.043^{*}$ \\
\hline
\end{tabular}

\section{Discussion}

The present work is, to the best of our knowledge, the first to evaluate the impact of long WLT on NURS rates. We found an independent, direct, and linear correlation between WLT and NURS. While the OR of 1.005 (95\% Cl 1.00-1.01) per day may be perceived as a weak association, the cumulative risk reached an OR of $2.18(\mathrm{p}=0.043)$ after 60 days; this result is relevant for centers with a long WLT of any cause.

Our results are consistent with previous research showing an increased risk of NURS in patients with small and radiolucent stones. Although the association of NURS with female gender and distal location did not reach statistical significance in multivariate analysis, there was a trend towards an increased risk that reached statistical significance in univariate analysis, congruent with prior reports.

\begin{tabular}{lcc}
\hline \multicolumn{3}{l}{ Table 3. Negative ureteroscopy rates per month } \\
\hline $\begin{array}{l}\text { Waiting-list time } \\
\text { (months) }\end{array}$ & $\begin{array}{c}\text { Negative URS/total } \\
\text { URS }\end{array}$ & $\begin{array}{c}\text { Negative } \\
\text { ureteroscopy rate }\end{array}$ \\
\hline 1 & $4 / 37$ & $10.8 \%$ \\
2 & $11 / 90$ & $12.2 \%$ \\
3 & $16 / 122$ & $13.1 \%$ \\
4 & $25 / 152$ & $16.4 \%$ \\
5 & $31 / 185$ & $16.7 \%$ \\
6 & $35 / 199$ & $17.5 \%$ \\
$>6$ & $41 / 219$ & $18.7 \%$ \\
\hline URS: ureteroscopy. & &
\end{tabular}




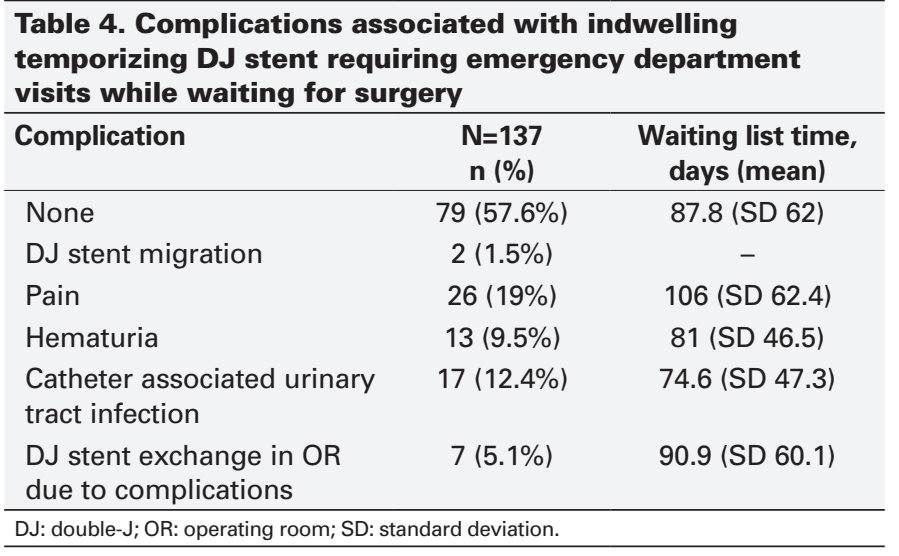

NURS are frustrating events for patients and urologists alike. Unnecessary surgical and anesthetic risks, hospital costs, and sick leave have personal, institutional, and social repercussions that should be avoided whenever possible.

A systematic review and meta-analysis of the cost of ureteroscopies, which included 12 studies (most of them from the U.S.), showed a mean overall cost of $\$ 2801$ for every URS, though the overall estimated cost was lower in other countries, such as China, Egypt, and the U.K. ${ }^{9}$ In regards to complications, a global study performed by the Clinical Research Office of the Endourological Society Ureteroscopy (CROES) with 11885 patients, of whom 8676 had only ureteral stones, found an overall postoperative complication rate of $3.5 \%$; fever was the most frequent complication $(1.8 \%)$, followed by urinary tract infection $(1 \%)$, bleeding $(0.4 \%)$, bladder cramps $(0.4 \%)$, and sepsis $(0.3 \%) .^{10}$

NURS rates reported in the literature range from $3.8-13 \% .^{1-5}$ Many authors have sought to determine the risk factors associated with NURS in order to avoid unnecessary surgical interventions.

Kreshover et al reported a NURS rate of $9.8 \%$ and found that small stone size (OR 0.55) and distal location (OR 2.5) increased the risk of NURS. ${ }^{3}$ Katafigiotis et al, in 2018, described a NURS rate of $3.8 \%$ and found a statistically significant association with female gender (OR 3.93, 95\% Cl 1.48-10.50, $\mathrm{p}=0.006)$, radiopacity at KUB X-ray (OR 9.57, 95\% Cl 2.54-36.09, $\mathrm{p}<0.001$ ), and stone surface area as measured on CT scan (OR 0.91, 95\% Cl 0.87-0.96, $\mathrm{p}<0.001) ;{ }^{1}$ however, the width of the confidence intervals reduces the certainty of most findings. Prattley et al found a NURS rate of $13 \%$ and identified stone size, distal location, and young age as showing a statistically significant association, but since no measure of association was reported, the strength of the association remained unknown, making it impossible to compare results. ${ }^{2}$

Sahin et al evaluated the impact of time from CT scan to surgery, finding a statistically significant difference (OR $1.193,95 \% \mathrm{Cl} 1.140-1.248, \mathrm{p} \leq 0.001)$. However, since the mean time in the PURS and NURS groups was 4.6 and
12.1 days, respectively, the impact of a long WLT remained unknown. ${ }^{5}$ They also found that BMI (OR 0.86, 95\% CI $0.782-0.953, p=0.004)$, distal location (OR 2.8, 95\% Cl $1.192-6.622, p=0.018$ ), stone surface area (OR 0.96, 95\% $\mathrm{Cl} 0.950-0.978, \mathrm{p} \leq 0.001)$, and medical expulsive therapy use (OR 4.2, 95\% Cl 1.829-9.877, $\mathrm{p}=0.001$ ) had a statistically significant association with NURS. ${ }^{5}$ These findings are similar to our series, where $58.5 \%(24 / 41)$ of the NURS patients had a stone size of $\leq 6 \mathrm{~mm}$ and $71.1 \%(30 / 41)$ had a distal location, which could serve as a cutoff.

Apart from the work by Katafigiotis et al, ${ }^{1}$ our study is the only series including patients with temporary DJ stents, and in contrast to those authors' findings, we did observe a statistically significant decreased risk of having a NURS (OR $0.43,95 \% \mathrm{Cl} 0.2-0.8, \mathrm{p}=0.019)$, meaning it makes the URS more likely to be positive.

One of the strengths of our cohort is that it included patients with indwelling temporary DJ stents. This renders our study more pragmatic and applicable to real-world settings, as previous studies have shown a spontaneous stone passage rate of $8-14 \%$ in patients with such stents. ${ }^{11,12}$ Given that in our multivariate analysis the presence of a temporary DJ stent was found to be protective against NURS, we consider it relevant to include these patients when analyzing NURS rates.

Among the limitations of our study is the fact that not all patients underwent a preoperative CT scan. This may have entailed a risk of reduced accuracy, bearing in mind that it has been reported that CT scan has a sensitivity of $95-100 \%$ and a specificity of $96-98 \%$ for the detection of ureteral stones, whereas KUB X-ray has a sensitivity of 59\% and a specificity of $71 \% .{ }^{13}$ However, in our institutional protocol, all patients underwent either a CT scan or both a KUB X-ray and urinary system ultrasound, with the sensitivity and specificity of this combination being reported to be $96 \%$ and $91 \%$, respectively. ${ }^{14}$

The accuracy of stone size measurement has been shown to be very similar between CT scan and KUB X-ray, with less than $1 \mathrm{~mm}$ of difference between the measurements, ${ }^{15}$ and although the accuracy of ultrasound for ureteral stone size measurement is arguably worse than that of CT or KUB, a recent study of 1289 patients with ureteral stones showed that if a stone is seen, there is a high correlation in size on ultrasound and CT scan. ${ }^{16}$ We therefore consider our data to be sufficiently precise to group together the size estimates from all tests.

Another limitation is the lack of a definition of what constitutes a long WLT in patients with non-malignant conditions. Different hospitals will have different interpretations of this, but considering that the natural history of conservatively managed ureteral stones shows spontaneous passage within four weeks, on average, ${ }^{17}$ and that spontaneous passage is also measured at four weeks in clinical trials, ${ }^{18}$ a cutoff of 
two months ( $\geq 60$ days) may be considered prudent. In our study, we observed that the rate of NURS in the first two months was similar to rates previously reported in the literature, ${ }^{1-5}$ while patients operated on beyond two months had an exceedingly high NURS rate.

Although there is no clear explanation as to why a long WLT may increase the NURS rate, one possible explanation is that radiolucent stones, likely to be composed at least partially of uric acid, may decrease in size due to urinary $\mathrm{pH}$ changes and pass spontaneously. We observed that radiolucent stones were more prevalent in the NURS group; in this context, it should be borne in mind that the natural history of non-obstructing uric acid stones is still unknown. ${ }^{19}$

The results of our study provide centers experiencing long WLTs (defined as $\geq 60$ days) with more tools to identify patients at higher risk of NURS. Such centers may consider developing protocols for repeated preoperative imaging with non-contrast $\mathrm{CT}$, particularly in patients with small, distal, radiolucent stones and with more than 60 days since the last imaging, regardless of the presence of an indwelling DJ stent.

\section{Conclusions}

We found that a long WLT is directly associated with exceedingly high NURS rates. Long surgical WLT is a frequent problem in high-volume centers, and such centers should consider developing protocols for the described subset of patients at the highest risk of NURS. Based on our findings, we suggest that patients with small (e.g., $\leq 6 \mathrm{~mm}$ ) radiolucent stones and with a long WLT ( $\geq 60$ days) may be offered CT re-evaluation before surgery, as up to $18.7 \%$ of these patients may already have passed the stone and could avoid an unnecessary procedure.

Competing interests: The authors do not report any competing personal or financial interests related to this work.

This paper has been peer-reviewed.

\section{References}

1. Katafigiotis I, Sabler IM, Heifetz EM, et al. "Stoneless" or negative ureteroscopy: A reality in the endourologic routine or avoidable source of frustration? Estimating the risk factors for a negative ureteroscopy. J Endourol 2018;32:825-30. https://doi.org/10.1089/end.2018.0291

2. Prattley S, Rice P, Pietropaolo A, et al. Predictors and results of negative ureteroscopy for treatment of consecutive ureteric stones done as a primary procedure: Prospective outcomes from a university hospital. Urol Int 2019;103:143-8. https://doi.org/10.1159/000501658

3. Kreshover JE, Dickstein RJ, Rowe C, et al. Predictors for negative ureteroscopy in the management of upper urinary tract stone disease. Urology 2011;78:748-52. https://doi.org/10.1016/i.uroogy.2011.03.008

4. Lamberts RW, Conti SL, Leppert JT, et al. Defining the rate of negative ureteroscopy in the general population treated for upper tract urinary stone disease. J Endourol 2017;31:266-71. https://doi.org/10.1089/end.2016.0751

5. Sahin $M O$, Sen V, Irer $B$, et al. Can negative ureteroscopy be predicted in ureteral stone treatment? Can Urol Assoc J 2020;14:E209-13. hitps://doi.org/10.5489/cuaj.6026

6. Lipkin M, Ackerman A. Imaging for urolithiasis: Standards, trends, and radiation exposure. Curr Opin Urol 2016;26:56-62. https://doi.org/10.1097/MOU.0000000000000241

7. Fröber R. Surgical anatomy of the ureter. BJU Int 2007;100:949-65. https://doi.org/10.1111/i.1464410X.2007.07207.x

8. von Elm $E$, Altman DG, Egger $M$, et al. Strengthening the reporting of observational studies in epidemiology (STROBE) statement: Guidelines for reporting observational studies. BMJ 2007;335:806-8. https://doi.org/10.1136/bmi.39335.541782.AD

9. Geraghty RM, Jones P, Herrmann TRW, et al. Ureteroscopy is more cost effective than shockwave lithotripsy for stone treatment: Systematic review and meta-analysis. World I Urol 2018;36:1783-93. https://doi.org/10.1007/s00345-018-2320-9

10. de la Rosette J, Denstedt J, Geavlete P, et al. The Clinical Research Office of the Endourological Society Ureteroscopy global study: Indications, complications, and outcomes in 11885 patients. J Endourol 2014;28:131-9. https://doi.org/10.1089/end.2013.0436

11. Baumgarten L, Desai A, Shipman S, et al. Spontaneous passage of ureteral stones in patients with indwelling ureteral stents. Can J Urol 2017;24:9024-9.

12. Kuebker JM, Robles J, Kramer JJ, et al. Predictors of spontaneous ureteral stone passage in the presence of an indwelling ureteral stent. Urolithiasis 2019;47:395-400. https://doi.org/10.1007/s00240018-1080-8

13. Levine JA, Neitlich J, Verga $M$, et al. Ureteral calculi in patients with flank pain: Correlation of plain radiography with unenhanced helical CT. Radiology 1997;204:27-31. https://doi.org/10.1148/ radiology.204.1.9205218

14. Mitterberger $M$, Pinggera $G M$, Pallwein $L$, et al. Plain abdominal radiography with transabdominal native tissue harmonic imaging ultrasonography vs. unenhanced computed tomography in renal colic. BJU Int 2007;100:887-90. https://doi.org/10.1111/i.1464-410X.2007.07048.x

15. Parsons JK, Lancini V, Shetye K, et al. Urinary stone size: Comparison of abdominal plain radiography and non-contrast CT measurements. J Endourol 2003;17:725-8. https://doi.org/10.1089/089277903770802245

16. Liu D, Wu J, Chen S, et al. Ultrasonography can replace CT scans as the initial imaging examination of ureteral calculi. Urol Int 2019;103:68-73. https://doi.org/10.1159/000500099

17. Yallappa $S$, Amer $T$, Jones $P$, et al. Natural history of conservatively managed ureteral stones: Analysis of 6600 patients. J Endourol 2018;32:371-9. htrps://doi.org/10.1089/end.2017.0848414

18. Pickard R, Starr K, MacLennan G, et al. Medical expulsive therapy in adults with ureteric colic: A multicenter, randomized, placebo-controlled trial. Lancet 2015;386:341-9. https://doi.org/10.1016/ S0140-6736(15)60933-3

19. Abou-Elela A. Epidemiology, pathophysiology, and management of uric acid urolithiasis: A narrative review. J Adv Res 2017;8:513-27. https://doi.org/10.1016/i.jare.2017.04.005

Correspondence: Dr. Daniel A. González-Padilla' Department of Urology, University Hospital Infanta Sofía, Madrid, Spain; daniel.mx@gmail.com 